\title{
Study on the Edge Fusing Technology
}

\author{
Lehui Huang ${ }^{a}$, Wenfang $\mathrm{Xu}^{\mathrm{b}}$ \\ Education School, Jiangxi Science\&Technology Normal University, Nanchang, China

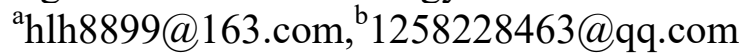

Keywords: Edge Blending; Seamless Splicing Technology; Visual Effects

\begin{abstract}
Edge blending technology can solve the problem of seamless splicing in large screen effectively and improve the visual effect of mosaic images. This paper will discuss the developing process of edge fusing technology, compare it with traditional projection splicing technology, introduce its field of application and predict its prospect.
\end{abstract}

\section{Introduction}

Edge blending technology means the display mode by using multiple projectors to display complete image on the entire screen according to the partition and the composition. The single physical grating of every projector form many "convergence zones" by means of overlapping parts of each other, and then reduce "extra" brightness of the fusing band through the raster feathering function of projector built-in or external device to produce a complete screen. In theory, no matter how large a screen is, one projector is enough for it. But if the screen is too large, the brightness per unit area on the screen is lower; in that case you need to use multiple projectors to "share" the display area to improve the screen brightness to make it reach the standards, that's why blending projection come into being.

\section{The developing process}

Edge blending technology is mainly to achieve the integration of multiple images through dealing with the edge of projection. From the aspects of fusing splicing effect, seamless splicing technology mainly experiences the following three development stages: hard edge stitching, overlapped stitching and edge fusion splicing.

1). Hard Edge stitching: This is the initial edge fusion technology; the edge of two projectors is aligned without overlapping sections. Hard edge stitching is simple to operate, but the effect is not very good and shows as the split complete picture by a seam. If brightness enhancement processing was not made at the projector edge, the seam appears black; otherwise the seam shows in white. In a word, hard edge stitching leaves significant dividing line (physical patchwork) and cannot achieve integrated panoramic display.

2). Overlap splicing: the two projector screen with some overlap area has no fade out treatment, so the brightness of the overlapping portion is 2 times of the rest of the whole picture. In the display part, the overlapping part is showed as a light bar. The image from two projectors overlaps in a superposition in the joint area, which will lead to over-bright zone due to brightness superimposing in the overlapping area and impact the achievement of seamless effect. 
3). Edge fusion splicing: Compared with simple overlapping methods, the brightness of overlapping right part of the left projector is weakened linearly, while the left part of the right projector enhanced linearly. When refers to the display result, the brightness of the whole picture is coincident totally. With the edge fusing technology, we can not only get complete fusion, but also eliminate the over light zone that caused by overlapping blending. What's more, the edge fusion splicing technology can be adapted to a variety of curved surface like plane, cylinder, sphere, etc, so it possess more extensive Applicability.

\section{The difference between the edge of fusion technology and traditional mosaic}

1). In conventional splicing systems, because of different production time and different production environments, there exist objective differences of thermal expansion and contraction on different screens. Even using the same material, the difference is difficult to avoid between various screens. It will cause that some minor physical changes will happen to the entire system after a period of applying, thereby affecting the performance and stability of the entire system. However, for applying an entire screen with the same level of technology, the same production time and the same material, the edge fusion technology can eliminate these problems above.

2). In fusion splicing, for the use of the entire screen, the physical gap between traditional splicing screens is eliminated, which maintains the screen image integrity and avoid artificial segmentation. And after using fusion technology, the optical gap defect is eliminated. Referring to the technical level and display effect, there is qualitative difference and improvement compared with ordinary reckless system: the integrity and aesthetics of display image is maintained, the image that displayed is concordant exactly without any physical or optical resolution. This is especially important when displaying the image information like maps, drawings, etc. Because there are a lot of lines or routes on drawings and maps, and the screen cracks and optic gaps will cause image display pollution, which will make the observer mistake easily the displayed image line staff and the original line of the splicing system, leading to mistake on decision-making and research. In fact, we can avoid this situation through integrating process.

3). In fusion splicing system, since all the images have been corrected and united by merging processor, so the brightness, color, evenness, vividness are more consistent regardless of the format of the image when displaying and switching image. So the displaying quality caused by replacing sign appearing in the traditional stitching systems frequently, will not arise.

\section{Main application areas}

1). Power Industry:

In order to create effective communications environment, all of the information collected must be promptly provided to the team members in control room, so the display wall system becomes the focus of the overall operation. All applications software is presented to the operator visual immediately, which requires large screen blending display to improve quality of image display and enhance the reactive efficiency of the operator.

2). Coal Mines, Transportation, Education and other monitoring systems:

Large-screen display system can be used to achieve interoperability with existing video conferencing, internal communications, information and data collection, early network warning, sea information monitoring, computer local area network, central command and information systems and other equipments. The use of computer technology, communication technology, and video surveillance technology can complete comprehensive and high-speed information processing, and achieve high-resolution, high brightness, multi-screen, multi-function display capability. And with the function of editing and storing pictures, it can make the various signals displayed on a large 
screen on the wall to make all situations obvious, so as to reach the goal of real-time monitoring and centralized control.

3). Telecom network operating center

At present, telecom network management center uses digital display video wall systems for telecom operators to provide integrated management of multiple networks and large-scale high-definition display, making it easy to operate with high intelligent control system. With powerful function of display image and combined windows of digital blending wall system, we can collect various of real-time information such as the interactive network, transmission network, signaling network, intelligent network, GSM network, GPRS network, and other real-time information including: digital maps, Wi-Fi base station maps, NE topology map, real-time network performance state table, real-time alerts table, statistical analysis table, and then converge them to the same combination to display in a screen. Sharing resources between systems can be realized independently each other, which enhance the cooperation, communication, and further improve unified management capabilities of network management center.

\section{Developing prospects}

With the progress of national economy, people pay more attention to high-performance edge converged device, and the requirements for ultra-high resolution image processing and the equipment performance are rising increasingly. With the expanding application scope of edge blending system, the single projection display has been unable to meet the display requirements of the exhibition, enterprises hall, and group meetings. Large visual electronic active stereo and edge blending passive stereo effect and show high-definition 3D effect has met the application of a variety of industry, even virtual simulation, interactive multimedia, large digital sand table display systems has reached professional level. Edge Blending gradually has rapid development in multi-screen stocks, corporate video promotion, event promotion, advertising media and other industries requiring excellent large screen, from the original several industries.

In the next three to five years, the use of projection edge blending will become the mainstream of the integration industry. The domestic growth momentum of HD fusion indicates that China will enter the era of high-definition projection convergence completely and the edge of fusion technology will create a new era. We can see large electronic case one by one settled in large-scale exhibition, high-definition video sites, and many college authorities. On the other hand, edge converged device begins to emerge in the business field from the outset more professional and high-end applications to a number of segments applications in business, entertainment, and public administration. Especially in several areas of the edge of fusion, it is widely used and stimulates the rapid development of the industry. Together with the recent improvement of network technology and the penetration of the market, the projector fusion benefits a lot, at the same time, it also accelerates the rapid conversion of information and creates good environment. High-performance edge converged devices will have a bigger and better stage to play its role in the future.

\section{Conclusion}

The development of edge blending technology adapts to the trend of the times and meets people's need of the big screen viewing, widely used in many fields of teaching, transportation, telecommunications and other network operators. With continuous improvement of the edge of fusion technology, the network technology, as well as the popularity of the market, projected fusion technology will have unlimited potential in the future development. 


\section{References}

[1] Li Bin. Method studies on low-cost multi-projector stitching [D].Shandong University, 2007.04.05.

[2] Sui Yonghe. On the development of edge of fusion technology systems principles[J].Theory of Urban Construction, 2012.

[3] Zhu Jimin. On the edge of the big screen seamless fusion splicing technology[J]. Chinese New Technology and New Products, 2010.10.10.

[4] Gao Guobao, Chai Haifeng. Problems of Edge fusion splicing projection[J]. Advanced Display, 2012.03.05.

[5] Yu Linyun, Wang Yigang. Seamless splicing technology based on software in large-screen projection system [J].Computer Simulation, 2009.05.15. 